Applied Mathematical Sciences, Vol. 8, 2014, no. 165, 8257 - 8269

HIKARI Ltd, www.m-hikari.com

http://dx.doi.org/10.12988/ams.2014.411913

\title{
Lower and Upper Bounds for Chord Power Integrals of Ellipsoids
}

\author{
Lothar Heinrich \\ Augsburg University, Institute of Mathematics \\ 86135 Augsburg, Germany \\ Dedicated to Professor Marius Stoka on the occasion of his $80^{\text {th }}$ birthday
}

Copyright (c) 2014 Lothar Heinrich. This is an open access article distributed under the Creative Commons Attribution License, which permits unrestricted use, distribution, and reproduction in any medium, provided the original work is properly cited.

\begin{abstract}
First we discuss different representations of chord power integrals $\mathcal{I}_{p}(K)$ of any order $p \geq 0$ for convex bodies $K \subset \mathbb{R}^{d}$ with inner points. Second we derive closed-term expressions of $\mathcal{I}_{p}(\mathbb{E}(\mathbf{a}))$ for an ellipsoid $\mathbb{E}(\mathbf{a})$ with semi-axes $\mathbf{a}=\left(a_{1}, \ldots, a_{d}\right)$ in terms of the support function of $\mathbb{E}(\mathbf{a})$ and prove upper and lower bounds expressed by the volume and the mean breadth of $\mathbb{E}(\mathbf{a})$, respectively. A further inequality conjectured in Davy (1984) is proved for ellipsoids. Some remarks on chord power integrals of superellipsoids and simplices round off the topic.
\end{abstract}

Mathematics Subject Classification: Primary 52A40 60G05 ; Secondary 52A07 52A22

Keywords: Poisson cylinder process, integral \& convex geometry, convex body, support function, mean breadth, isoperimetric \& Carleman's inequality

\section{Chord Power Integrals - Definition and Basics}

Let $K$ be a convex body in $\mathbb{R}^{d}$ with interior points and $\mathbb{S}^{d-1}=\partial \mathbb{B}^{d}$ the boundary of the Euclidean unit ball $\mathbb{B}^{d}=\left\{\mathbf{x} \in \mathbb{R}^{d}:\|\mathbf{x}\| \leq 1\right\}$. Further, let $\mathcal{H}^{k}$ denote 
the $k$-dimensional Hausdorff measure on $\mathbb{R}^{d}$ for $k=1, \ldots, d$. As usual let us denote by $V(K)=\mathcal{H}^{d}(K)$ and $S(K)=\mathcal{H}^{d-1}(\partial K)$ volume and surface content of $K$, respectively. Further, we recall that $\kappa_{d}:=V\left(\mathbb{B}^{d}\right)=\pi^{d / 2} / \Gamma(d / 2+1)$ and $S\left(\mathbb{B}^{d}\right)=d \kappa_{d}$ with $\Gamma(s):=\int_{0}^{\infty} e^{x} x^{s-1} \mathrm{~d} x$ for $s>0$.

For any $p \geq 0$ we define the pth-order chord power integral (CPI) of $K$ by

$$
\mathcal{I}_{p}(K)=\frac{1}{2} \int_{\mathbb{S}^{d-1}} \int_{K \mid \mathbf{u}^{\perp}}\left(\mathcal{H}^{1}(K \cap \ell(\mathbf{x}, \mathbf{u}))\right)^{p} \mathrm{~d} \mathbf{x} \mathcal{H}^{d-1}(\mathrm{~d} \mathbf{u})
$$

(with $0^{0}:=0$ ), where $\ell(\mathbf{x}, \mathbf{u}):=\{\mathbf{x}+\alpha \mathbf{u}: \alpha \in \mathbb{R}\}$ stands for the line in direction $\mathbf{u} \in \mathbb{S}^{d-1}$ through $\mathbf{x} \in \mathbb{R}^{d}$ and $K \mid \mathbf{u}^{\perp}$ is the orthogonal projection of $K$ on $\mathbf{u}^{\perp}(=(d-1)$-dimensional subspace orthogonal to $\mathbf{u})$. CPI's are of considerable interest in integral and stochastic geometry for a long time, see [11], [13], and have many applications in material sciences, physics and image analysis, see e.g. [4], [5], [14] and references therein. In textbooks of integral and convex geometry, see e.g. [11], [13], the r.h.s. of (1) is mostly written as integral w.r.t. the line measure $\mu_{1}^{(d)}(\cdot)$ (defined on the space $\mathbb{A}(d, 1)$ of one-dimensional affine subspaces of $\mathbb{R}^{d}$ ):

$$
\mathcal{I}_{p}(K)=\frac{d \kappa_{d}}{2} \int_{\mathbb{A}(d, 1)}\left(\mathcal{H}^{1}(K \cap L)\right)^{p} \mu_{1}^{(d)}(\mathrm{d} L),
$$

where, for integers $p=2, \ldots, d$, the Blaschke-Petkantschin formula, see [11] (p. 363) provides the representations

$$
\mathcal{I}_{k+1}(K)=\frac{(k+1) d \kappa_{d}}{2 \kappa_{k}} \int_{\mathbb{A}(d, k)}\left(\mathcal{H}^{k}(K \cap L)\right)^{2} \mu_{k}^{(d)}(\mathrm{d} L)
$$

for $k=1, \ldots, d$ with the motion-invariant $k$-flat measure $\mu_{k}^{(d)}(\cdot)$ (defined on the space $\mathbb{A}(d, k)$ of $k$-dimensional affine subspaces of $\left.\mathbb{R}^{d}\right)$ satisfying the normalization $\mu_{k}^{(d)}\left(\left\{E \in \mathbb{A}(d, k): E \cap \mathbb{B}^{d} \neq \emptyset\right\}\right)=\kappa_{d-k}$. From (1) for $p=0,1$ and (3) for $k=d-1$ we get the following relations, see e.g. [12],

$$
\mathcal{I}_{0}(K)=\frac{\kappa_{d-1}}{2} S(K), \mathcal{I}_{1}(K)=\frac{d \kappa_{d}}{2} V(K), \mathcal{I}_{d+1}(K)=\frac{d(d+1)}{2} V(K)^{2} .
$$

Remark 1. The moments $m_{k}(K):=\mathcal{I}_{k}(K) / \mathcal{I}_{0}(K)=\mathrm{E} L_{\mu, K}^{k}$ for $k=1,2, \ldots$ determine a unique distribution function $F_{\mu, K}$ (of the length $L_{\mu, K}$ of the $\mu$ random chord of $K$ ) which, however, does not characterize the shape of $K$ completely, see [11].

Due to F. Piefke [9], see also [12], the r.h.s. of (1) can be expressed for any $p>1$ by the distribution of the interpoint distance of two randomly chosen 
points inside $K$ leading to

$$
\frac{2 \mathcal{I}_{p}(K)}{p(p-1)}=\int_{K} \int_{K} \frac{\mathrm{d} \mathbf{x} \mathrm{d} \mathbf{y}}{\|\mathbf{x}-\mathbf{y}\|^{d+1-p}}=\int_{K \oplus(-K)} \frac{V(K \cap(K+\mathbf{x})) \mathrm{d} \mathbf{x}}{\|\mathbf{x}\|^{d+1-p}}
$$

for any real $p>1$, i.e., the ratio $\mathcal{I}_{p}(K) / V(K)^{2}$ takes the form

$$
\frac{\mathcal{I}_{p}(K)}{V(K)^{2}}=\frac{p(p-1)}{2} \int_{\mathbb{R}^{d}} \int_{\mathbb{R}^{d}} \frac{U_{K}(\mathrm{~d} \mathbf{x}) U_{K}(\mathrm{~d} \mathbf{y})}{\|\mathbf{x}-\mathbf{y}\|^{d+1-p}} \text { with } U_{K}(\cdot)=\frac{\mathcal{H}^{d}((\cdot) \cap K)}{\mathcal{H}^{d}(K)}
$$

and the integral on r.h.s of the last line is known as $(d+1-p)$-energy of the probability measure $U_{K}(=$ uniform distribution on $K)$.

\section{CLT for a Class Poisson Cylinder Processes}

To motivate our study of CPI's we state a central limit theorem (CLT) for the total volume of the union of isotropic Poisson $k$-cylinders included in an expanding convex domain $\varrho K$ as $\varrho \uparrow \infty$, where $K \subset \mathbb{R}^{d}$ is a convex body containing the origin o of $\mathbb{R}^{d}$ as inner point. To be precise we need some further notation. For details and the proof of the below CLT the reader is referred to [7].

Let $\Pi_{\lambda}=\left\{P_{i}\right\}_{i \geq 1}$ be a stationary Poisson point process on $\mathbb{R}^{d-k}$ with positive intensity $\lambda:=\mathrm{E} \#\left\{i \geq 1: P_{i} \in[0,1]^{d-k}\right\}$, where each point $P_{i}$ is associated with an independent copy $\left(\Xi_{i}, \Theta_{i}\right)$ of some generic pair $\left(\Xi_{0}, \Theta_{0}\right)$ which consists of a random compact set $\Xi_{0} \subset \mathbb{R}^{d-k}$ satisfying $0<E \mathcal{H}^{d-k}\left(\Xi_{0}\right)^{2}<\infty$ and a random orthogonal matrix $\Theta_{0}$ whose (uniform) distribution is induced by the normalized Haar measure on the Grassmannian of $k$-dimensional subspaces in $\mathbb{R}^{d}$. In addition, the components $\Xi_{0}$ and $\Theta_{0}$ are independent and the sequence $\left\{\left(\Xi_{i}, \Theta_{i}\right)\right\}_{i \geq 1}$ is generated stochastically independent of $\Pi_{\lambda}$.

The countable familiy of random closed sets in $\mathbb{R}^{d}$

$$
\Theta_{i}\left(\left(\Xi_{i} \oplus P_{i}\right) \times \mathbb{R}^{k}\right):=\left\{\Theta_{i}\left(\xi+P_{i}, \mathbf{x}\right) \in \mathbb{R}^{d}: \xi \in \Xi_{i}, \mathbf{x} \in \mathbb{R}^{k}\right\} \text { for } i \geq 1
$$

forms a stationary and isotropic process of Poisson $k$-cylinders in $\mathbb{R}^{d}$ for $k=$ $1, \ldots, d-1$. The motion-invariant union set of the Poisson $k$-cylinders

$$
\Xi_{d, k}:=\bigcup_{i \geq 1} \Theta_{i}\left(\left(\Xi_{i} \oplus P_{i}\right) \times \mathbb{R}^{k}\right) \text { for each } k=1, \ldots, d-1
$$

is observed in an unboundedly increasing convex window $\varrho K$ as $\varrho \uparrow \infty$, see Fig. 1 and Fig. 2 for realizations of $\Xi_{2,1}$ and $\Xi_{3,1}$ with $K=[0,1]^{2}$ and $K=$ $[0,1]^{3}$, respectively. 
Now we are in a position to formulate the announced CLT for the volume fraction of $\Xi_{d, k}$ in $\varrho K$ for $k=1, \ldots, d-1$ : As $\varrho \uparrow \infty$, the random variable

$$
\varrho^{(d-k) / 2}\left(\frac{\mathcal{H}^{d}\left(\Xi_{d, k} \cap \varrho K\right)}{\varrho^{d} V(K)}-\mathrm{EH}^{d}\left(\Xi_{d, k} \cap[0,1]^{d}\right)\right)
$$

is asymptotically normally distributed with mean zero and variance

$$
\sigma^{2}(K)=\frac{2 \kappa_{k} \lambda M_{2} e^{-2 \lambda M_{1}}}{(k+1) d \kappa_{d}} \frac{\mathcal{I}_{k+1}(K)}{V(K)^{2}},
$$

where $\mathrm{EH}^{d}\left(\Xi_{d, k} \cap[0,1]^{d}\right)=1-e^{-\lambda M_{1}}$ with $M_{j}:=\mathrm{E} \mathcal{H}^{d-k}\left(\Xi_{0}\right)^{j}, j=1,2$.

This CLT is of interest from several points of view. The Gaussian limit of (5) depends on the shape of the observation window, i.e. on $K$, expressed in terms of the CPI $\mathcal{I}_{k+1}(K)$. This shape-dependence of $\sigma^{2}(K)$ is caused by the intrinsic long-range correlations of the random set $\Xi_{d, k}$. In contrast to this, in case of random sets satisfying certain weak dependence conditions, e.g. as in the degenerate case $k=0$, the asymptotic variance $\sigma^{2}(K)$ depends only on the volume $V(K)$. Note that $\Xi_{d, 0}$ can be identified with a stationary Boolean model with typical grain $\Xi_{0}$, see [13] (p. 117).

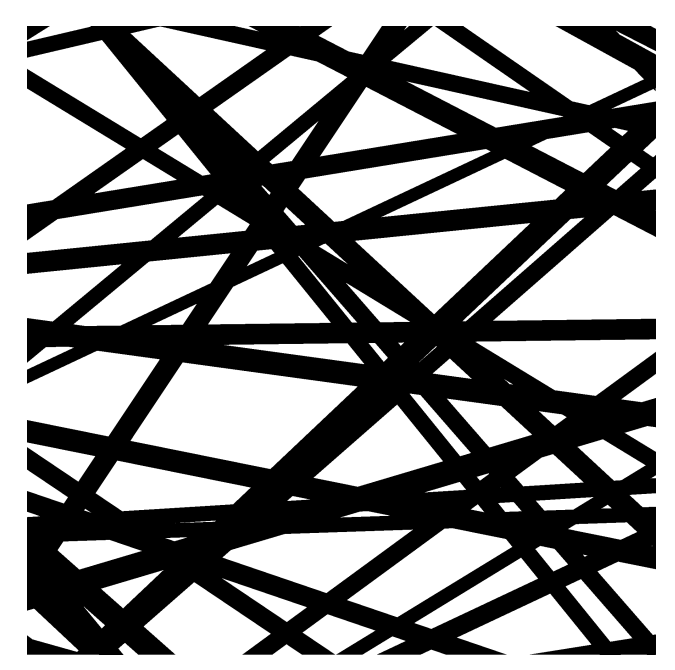

Figure 1: Isotropic Poisson strips

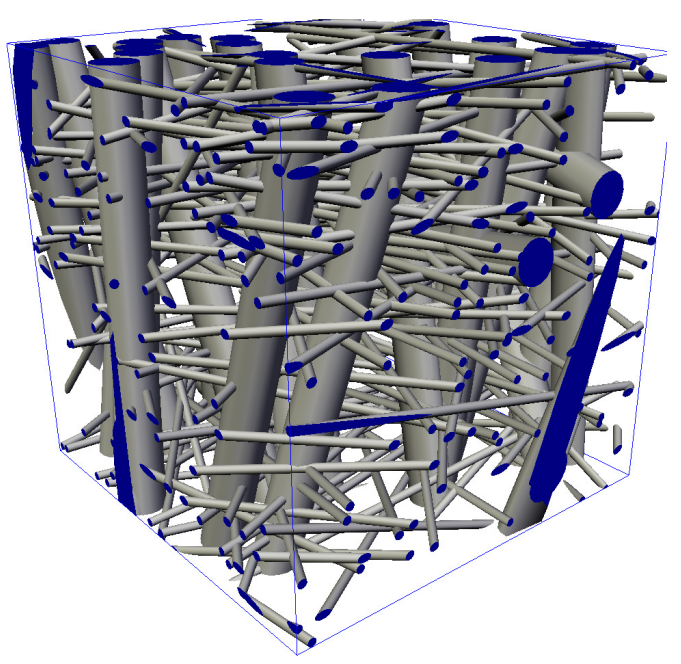

Figure 2: Poisson cylinders in a cube

Statisticians aim at designing observation procedures such that estimators of model characteristics have minimal variances. In our model this means to minimize the ratio $\mathcal{I}_{k+1}(K) / V(K)^{2}$ in (6) if another ovoid functional of $K$, e.g. the mean breadth $b(K)$, is fixed. In the planar case optimal lower bounds of $\mathcal{I}_{2}(K) / \mathcal{H}^{2}(K)^{2}$ have been obtained for particular classes of convex sets in [6] when the perimeter $\mathcal{H}^{1}(\partial K)$ is given. In convex geometry, see [3] or [13], 
one is also interested to maximize $\mathcal{I}_{k+1}(K)$ when $V(K)$ is fixed. Among all convex bodies the ball with radius $\left(V(K) / \kappa_{d}\right)^{1 / d}$ is the unique maximizer due to Carleman's inequality

$$
\mathcal{I}_{k+1}(K) \leq \frac{2^{k} d \kappa_{d} \kappa_{d+k}}{\kappa_{k+1}}\left(\frac{V(K)}{\kappa_{d}}\right)^{(d+k) / d} \text { for } k=0,1, \ldots, d
$$

In the main part of the paper we study CPI's of $d$-dimensional ellipsoids $\mathbb{E}(\mathbf{a})$ with positive semi-axes $\mathbf{a}=\left(a_{1}, \ldots, a_{d}\right)$ defined by

$$
\mathbb{E}(\mathbf{a})=\left\{\mathbf{x}=\left(x_{1}, \ldots, x_{d}\right): x_{1}^{2} / a_{1}^{2}+\cdots+x_{d}^{2} / a_{d}^{2} \leq 1\right\}
$$

\section{A Formula for CPI's of Ellipsoids}

From (8) it is easily seen that the diagonal matrix $A=\operatorname{diag}\left[a_{1}, \ldots, a_{d}\right]$ maps the unit ball $\mathbb{B}^{d}$ onto $\mathbb{E}(\mathbf{a})$, i.e. $\mathbb{E}(\mathbf{a})=A \mathbb{B}^{d}$, which implies the well-known formula $V(\mathbb{E}(\mathbf{a}))=\kappa_{d} \operatorname{det}(A)=\kappa_{d} a_{1} \cdots a_{d}$. The other intrinsic volumes $V_{j}(K), j=1, \ldots, d-1$, of the convex body $K$, see [13] (p. 600), with twice continuously differentiable boundary $\partial K$ can be expressed by the integral $\int_{\mathbb{S}^{d-1}} D_{j}\left(H_{K}(\mathbf{u})\right) \mathcal{H}^{d-1}(\mathrm{~d} \mathbf{u}) /(d-j) \kappa_{d-j}$, see [1]. In the latter formula the integrand is the sum of the $\left(\begin{array}{c}d-1 \\ j\end{array}\right)$ principal $j$-minors of the Hessian matrix $H_{K}(\mathbf{u}):=\left(\partial^{2} h_{K}(\mathbf{u}) / \partial u_{i} \partial u_{j}\right)_{i, j=1}^{d}$ of the support function $h_{K}(\mathbf{u}):=\max \{\langle\mathbf{u}, \mathbf{x}\rangle:$ $\mathbf{x} \in K\}$ of $K$ for $\mathbf{u}=\left(u_{1}, \ldots, u_{d}\right) \in \mathbb{S}^{d-1}$, where $\langle\mathbf{u}, \mathbf{x}\rangle=u_{1} x_{1}+\cdots+u_{d} x_{d}$. Basic facts on support functions and their role in convex geometry can be found in [1] and [13]. For example, the mean breadth $b(K)=2 \kappa_{d-1} V_{1}(K) / d \kappa_{d}$ and $S(K)=2 V_{d-1}(K)$ can be written in terms of $h_{K}(\cdot)$ as follows:

$$
b(K)=\frac{2}{d \kappa_{d}} \int_{\mathbb{S}^{d-1}} h_{K}(\mathbf{u}) \mathcal{H}^{d-1}(\mathrm{~d} \mathbf{u}) \quad, \quad S(K)=\int_{\mathbb{S}^{d-1}} D_{d-1}\left(H_{K}(\mathbf{u})\right) \mathcal{H}^{d-1}(\mathrm{~d} \mathbf{u}) .
$$

The support function of the ellipsoid $\mathbb{E}(\mathbf{a})$ defined in (8) is well-known and $D_{d-1}\left(H_{\mathbb{E}(\mathbf{a})}(\mathbf{u})\right)$ can be expressed (after rather lengthy calculations) by some power of $h_{\mathbb{E}(\mathbf{a})}(\mathbf{u})$ for $\mathbf{u} \in \mathbb{S}^{d-1}$, more precisely,

$$
h_{\mathbb{E}(\mathbf{a})}(\mathbf{u})=\sqrt{a_{1}^{2} u_{1}^{2}+\cdots+a_{d}^{2} u_{d}^{2}} \text { and } D_{d-1}\left(H_{\mathbb{E}(\mathbf{a})}(\mathbf{u})\right)=\frac{a_{1}^{2} \cdots a_{d}^{2}}{h_{\mathbb{E}(\mathbf{a})}(\mathbf{u})^{d+1}}
$$

This yields an integral expression of $\mathcal{I}_{0}(\mathbb{E}(\mathbf{a}))=\kappa_{d-1} S(\mathbb{E}(\mathbf{a})) / 2$. The remaining CPI's of $\mathbb{E}(\mathbf{a})$ are given in 
Theorem 3.1 For any real $p \geq 0$, we have

$$
\frac{\mathcal{I}_{p}(\mathbb{E}(\mathbf{a}))}{V^{2}(\mathbb{E}(\mathbf{a}))}=\frac{2^{p-1} \Gamma^{2}\left(\frac{d}{2}+1\right) \Gamma\left(\frac{p}{2}+1\right)}{\pi^{(d+1) / 2} \Gamma\left(\frac{d+p+1}{2}\right)} \int_{\mathbb{S}^{d-1}} \frac{\mathcal{H}^{d-1}(\mathrm{~d} \mathbf{u})}{h_{\mathbb{E}(\mathbf{a})}(\mathbf{u})^{d+1-p}}
$$

Proof. To begin with we rewrite (4) with indicator functions w.r.t. the ellipsoid $K=\mathbb{E}(\mathbf{a})$ so that, for any $p>1$,

$$
\frac{2 \mathcal{I}_{p}(\mathbb{E}(\mathbf{a}))}{p(p-1)}=\int_{\mathbb{R}^{d}} \int_{\mathbb{R}^{d}} \frac{\mathbf{1}_{\mathbb{E}(\mathbf{a})}(\mathbf{x}) \mathbf{1}_{\mathbb{E}(\mathbf{a})}(\mathbf{y}) \mathrm{d} \mathbf{x} \mathrm{d} \mathbf{y}}{\|\mathbf{x}-\mathbf{y}\|^{d+1-p}}
$$

By substituting $\mathbf{x}=A \mathbf{s}$ and $\mathbf{y}=A \mathbf{t}$ for $\mathbf{s}, \mathbf{t} \in \mathbb{B}^{d}$ and applying the integral transformation formula twice the double integral on the r.h.s. of (11) takes the form

$$
\prod_{i=1}^{d} a_{i}^{2} \int_{\mathbb{R}^{d}} \int_{\mathbb{R}^{d}} \frac{\mathbf{1}_{\mathbb{B}^{d}}(\mathbf{s}) \mathbf{1}_{\mathbb{B}^{d}}(\mathbf{t}) \mathrm{d} \mathbf{s} \mathrm{d} \mathbf{t}}{\|A(\mathbf{s}-\mathbf{t})\|^{d+1-p}}=\frac{V(\mathbb{E}(\mathbf{a}))^{2}}{\kappa_{d}^{2}} \int_{\mathbb{R}^{d}} \frac{V\left(\mathbb{B}^{d} \cap\left(\mathbb{B}^{d}+\mathbf{z}\right)\right) \mathrm{d} \mathbf{z}}{\|A \mathbf{z}\|^{d+1-p}} .
$$

Next, we introduce spherical coordinates $\mathbf{z}=r \mathbf{u}$ for $r=\|\mathbf{z}\| \geq 0$ and $\mathbf{u}=$ $\left(u_{1}, \ldots, u_{d}\right) \in \mathbb{S}^{d-1}$. Since the covariogram $V\left(\mathbb{B}^{d} \cap\left(\mathbb{B}^{d}+r \mathbf{u}\right)\right)=V\left(\mathbb{B}^{d} \cap\right.$ $\left.\left(\mathbb{B}^{d}+(r, 0, \cdots, 0)\right)\right)=: g_{\mathbb{B}^{d}}(r)$ of the unit ball depends only on $r \geq 0$ and disappears for $r>2$ we find together with the infinitesimal transformation rule $\mathrm{d} \mathbf{z}=r^{d-1} \mathrm{~d} r \mathcal{H}^{d-1}(\mathrm{~d} \mathbf{u})$ and $\|A \mathbf{z}\|=r\|A \mathbf{u}\|=r h_{\mathbb{E}(\mathbf{a})}(\mathbf{u})$ that

$$
\begin{aligned}
\frac{\mathcal{I}_{p}(\mathbb{E}(\mathbf{a}))}{V(\mathbb{E}(\mathbf{a}))^{2}} & =\frac{p(p-1)}{2 \kappa_{d}^{2}} \int_{0}^{2} \int_{\mathbb{S}^{d-1}} \frac{g_{\mathbb{B}^{d}}(r) r^{d-1} \mathcal{H}^{d-1}(\mathrm{~d} \mathbf{u}) \mathrm{d} r}{\|r A \mathbf{u}\|^{d+1-p}} \\
& =\frac{p(p-1)}{2 \kappa_{d}^{2}} \int_{0}^{2} g_{\mathbb{B}^{d}}(r) r^{p-2} \mathrm{~d} r \int_{\mathbb{S}^{d-1}} \frac{\mathcal{H}^{d-1}(\mathrm{~d} \mathbf{u})}{h_{\mathbb{E}(\mathbf{a})}(\mathbf{u})^{d+1-p}}
\end{aligned}
$$

It remains to express the first integral in the last line by values of the $\Gamma$ function. Arguing from a purely geometric view point (including Cavalieri's principle), see [6] (p. 326), we find that

$$
\begin{aligned}
& \int_{0}^{2} g_{\mathbb{B}^{d}}(r) r^{p-2} \mathrm{~d} r=2 \kappa_{d-1} \int_{0}^{2} \int_{0}^{r / 2}\left(\sqrt{1-x^{2}}\right)^{d-1} \mathrm{~d} x r^{p-2} \mathrm{~d} r \\
= & \frac{2^{p-1} \kappa_{d-1}}{p-1} \int_{0}^{1} r^{p / 2-1}(1-r)^{(d+1) / 2-1} \mathrm{~d} r=\frac{2^{p-1} \kappa_{d-1}}{p-1} \mathrm{~B}\left(\frac{p}{2}, \frac{d+1}{2}\right)
\end{aligned}
$$


with Euler's Beta-function $\mathrm{B}(a, b)$ satisfying $\mathrm{B}(a, b)=\Gamma(a) \Gamma(b) / \Gamma(a+b)$.

Summarizing the foregoing steps confirms (10) for $p>1$. The case $p \in[0,1]$ must be considered separately. From a formal point of view the term $p-1$ occurring in the above formulas disappears by cancelling and in view of the relation $\Gamma(p / 2) p / 2=\Gamma(p / 2+1)$ the r.h.s. of (10) makes sense for $p=0$ (and even for $p>-2$ ). To be rigorous we consider the r.h.s.'s of (1) and (10) as function of $p$ on the open interval $(0,1+\epsilon)$ for $\epsilon>0$. It can be shown that both integrals are analytic functions on this interval and right-continuous in $p=0$. Hence, both functions coincide and (10) holds for any $p \geq 0$.

\section{Alternative Calculation of CPI's for Ellipses}

Another way to calculate $\mathcal{I}_{p}(\mathbb{E}(\mathbf{a}))$ consists in a direct evaluation of the double integral (1). For ellipses $\mathbb{E}(a, b)=\left\{(x, y): x^{2} / a^{2}+y^{2} / b^{2} \leq 1\right\}$ with $a \geq b>0$ we will sketch this. For this purpose let $\mathbf{u}(\varphi)=(\cos \varphi, \sin \varphi)$ and assume w.l.o.g. that $0 \leq \varphi \leq \pi / 2$. To facilitate the integration over the projection interval $\mathbb{E}(a, b) \mid \mathbf{u}(\varphi)^{\perp}$ we rotate $\mathbf{u}(\varphi)$ and $\mathbb{E}(a, b)$ anti-clockwise by the angle $\hat{\varphi}:=\pi / 2-\varphi$. The rotation matrix $R(\varphi)=\left(r_{i j}\right)_{i, j=1}^{2}$ with $r_{11}=-r_{22}=\sin \vartheta, r_{21}=-r_{12}=\cos \vartheta$ and the support functions $h(\varphi)=$ $\sqrt{a^{2} \cos ^{2} \varphi+b^{2} \sin ^{2} \varphi}$ and $h(\hat{\varphi})=\sqrt{a^{2} \sin ^{2} \varphi+b^{2} \cos ^{2} \varphi}$ enables us to express the chord length in direction $\mathbf{u}_{0}=(0,1)$ of the rotated ellipse

$$
R(\varphi) \mathbb{E}(a, b)=\left\{(x, y): x^{2} h^{2}(\varphi)+y^{2} h^{2}(\hat{\varphi})-\sin (2 \varphi)\left(a^{2}-b^{2}\right) x y \leq a^{2} b^{2}\right\}
$$

as follows: $\quad \mathcal{H}^{1}\left(R(\varphi) \mathbb{E}(a, b) \cap \ell\left(x, \mathbf{u}_{0}\right)\right)=2 a b \sqrt{h^{2}(\hat{\varphi})-x^{2}} / h^{2}(\hat{\varphi})$

$$
\text { for } x \in[-h(\hat{\varphi}), h(\hat{\varphi})] \text {. }
$$

For reasons of symmetry we can reduce the integral over $\mathbb{S}^{1}$ on the r.h.s. of (1) to four integrals over the quadrant $[0, \pi / 2]$ leading to

$$
\mathcal{I}_{p}(\mathbb{E}(a, b))=2 \int_{0}^{\pi / 2} \int_{-h(\hat{\varphi})}^{h(\hat{\varphi})}\left(\frac{2 a b}{h(\hat{\varphi})}\left(1-\frac{x^{2}}{h^{2}(\hat{\varphi})}\right)^{1 / 2}\right)^{p} \mathrm{~d} \varphi .
$$

After a short calculation, where $h(\hat{\varphi})$ can be replaced by $h(\varphi)$, we arrive at

$$
\mathcal{I}_{p}(\mathbb{E}(a, b))=2^{p+1} \sqrt{\pi}(a b)^{p} \frac{\Gamma\left(\frac{p+2}{2}\right)}{\Gamma\left(\frac{p+3}{2}\right)} \int_{0}^{\pi / 2} \frac{\mathrm{d} \varphi}{h(\varphi)^{p-1}} \text { for any } p \geq 0
$$

It should be noted that (12) was given (without proof) in [14] for $p=0,1, \ldots$, see also [6] for $p=2$, and the chord length distribution function $F_{\mu, \mathbb{E}(a, b)}$ has been derived in [10]. Equating (10) for $d=2$ and (12) provides relations for complete elliptic integrals which are of interest in their own right. 
Finally, we say a few words in an attempt to use (3) to calculate $\mathcal{I}_{k+1}(\mathbb{E}(\mathbf{a}))$. For doing this, we need formulas for the $k$-volume of the intersection $k$-ellipsoids $\mathbb{E}(\mathbf{a}) \cap L$ for $L \in \mathbb{A}(d, k)$. For $k=d-1$ we succeeded in obtaining such a formula by exploiting the fact that the $(d-1)$-ellipsoids $E(p, \mathbf{u}):=\mathbb{E}(\mathbf{a}) \cap\left(\mathbf{u}^{\perp}+p \mathbf{u}\right)$ are homothetic for distinct $p \in\left[-h_{\mathbb{E}(\mathbf{a})}(\mathbf{u}), h_{\mathbb{E}(\mathbf{a})}(\mathbf{u})\right]$. Finally, after some further rearrangements we arrive at

$$
\mathcal{H}^{d-1}(E(p, \mathbf{u}))=\frac{\kappa_{d-1} V(\mathbb{E}(\mathbf{a}))}{\kappa_{d} h_{\mathbb{E}(\mathbf{a})}(\mathbf{u})}\left(1-\frac{p^{2}}{h_{\mathbb{E}(\mathbf{a})}(\mathbf{u})^{2}}\right)^{(d-1) / 2} .
$$

This inserted in (3) for $k=d-1$ shows that $\mathcal{I}_{d}(\mathbb{E}(\mathbf{a})) / V(\mathbb{E}(\mathbf{a}))^{2}$ is equal to

$$
\int_{\mathbb{S}^{d-1}} \int_{-h_{\mathbb{E}(\mathbf{a})}(\mathbf{u})}^{h_{\mathbb{E}(\mathbf{a})}(\mathbf{u})} \frac{\mathcal{H}^{d-1}(E(p, \mathbf{u}))^{2} \mathrm{~d} p}{(d-1) \kappa_{d-1} V(\mathbb{E}(\mathbf{a}))^{2}} \mathcal{H}^{d-1}(\mathrm{~d} \mathbf{u})=\frac{2^{d-1} \kappa_{2 d-1}}{\kappa_{d}^{3}} \int_{\mathbb{S}^{d-1}} \frac{\mathcal{H}^{d-1}(\mathrm{~d} u)}{h_{\mathbb{E}(\mathbf{a})}(\mathbf{u})} .
$$

\section{Some Sharp Estimates for CPI's of Ellipsoids}

Hölder's inequality applied to the moments $\mathrm{E} L_{\mu, K}^{k}$ in Remark 1 implies that

$$
m_{k+1}(K) \geq m_{i}(K) m_{j}(K) \geq m_{1}(K)^{k+1}=\left(d \kappa_{d} V(K) / \kappa_{d-1} S(K)\right)^{k+1}
$$

for $i+j=k+1$ and $k=0,1, \ldots$. Putting $i=1, j=k$ gives the inequality

$$
\frac{\mathcal{I}_{k+1}(K)}{\mathcal{I}_{k}(K)} \geq \frac{\mathcal{I}_{1}(K)}{\mathcal{I}_{0}(K)}=\frac{d \kappa_{d} V(K)}{\kappa_{d-1} S(K)} \text { for } k=0,1, \ldots, \text { see [11] (p. 48), }
$$

where "=" on the 1.h.s. is impossible for $k \geq 1$ since $\mathrm{P}\left(L_{\mu, K}=\right.$ const $)<1$.

P.J. Davy [3] posed the following considerable improvement of (13) as unsolved question. Just for $k \in\{d, d+1\}$ she gave a positive answer in [3].

Conjecture 1. For any convex body $K \subset \mathbb{R}^{d}$ with inner points and $k \geq 0$,

$$
\frac{\mathcal{I}_{k+1}(K)}{\mathcal{I}_{k}(K)} \geq \frac{2 \kappa_{k} \kappa_{d-1} \kappa_{d+k}}{\kappa_{k+1} \kappa_{d} \kappa_{d+k-1}} \frac{\mathcal{I}_{1}(K)}{\mathcal{I}_{0}(K)}=\frac{2 d \kappa_{k} \kappa_{d+k}}{\kappa_{k+1} \kappa_{d+k-1}} \frac{V(K)}{S(K)}
$$

with "=" being attained only for balls, where $\frac{2 \kappa_{k} \kappa_{d-1} \kappa_{d+k}}{\kappa_{k+1} \kappa_{d} \kappa_{d+k-1}}>1$ for $k \geq 1$.

To get an explicit lower bound of $\mathcal{I}_{k+1}(K)$ (as conjecture) we multiply on both sides of (14) over $k=1, \ldots, n$ (after that we set $n=k$ ) and replace $\mathcal{I}_{1}(K)$ by $d \kappa_{d} V(K) / 2$. In this way together with (7) we get the inclusion

$$
2^{k} d^{k+1} \frac{\kappa_{d+k}}{\kappa_{k+1}} \frac{V(K)^{k-1}}{S(K)^{k}} \leq \frac{\mathcal{I}_{k+1}(K)}{V(K)^{2}} \leq \frac{2^{k} d \kappa_{d+k}}{\kappa_{k+1} V(K)}\left(\frac{V(K)}{\kappa_{d}}\right)^{k / d}
$$


for $k=1, \ldots, d$, where for $k=0$ both sides of (15) are trivially true; the lower bound is proved for $k=d, d+1$, but still open for $k=1, \ldots, d-1$.

It should be noticed the fact that the inequality induced by the lower and upper bound in (15) coincides with the well-known isoperimetric inequality

$$
d \frac{V(K)}{S(K)} \leq\left(\frac{V(K)}{\kappa_{d}}\right)^{1 / d}, \quad \text { where " }=" \text { holds iff } K \text { is a ball. }
$$

This means that the validity of (15) would present an (almost) optimal estimate of $\mathcal{I}_{k+1}(K)$ and slightly strengthen the isoperimetric inequality.

In what follows we prove the inclusion (15) for $K=\mathbb{E}(\mathbf{a})$ and derive in this case at least for $k=d-1$ slightly larger lower bound in terms of $b(\mathbb{E}(\mathbf{a}))$.

Theorem 5.1 For the ellipsoid $K=\mathbb{E}(\mathbf{a})$ defined in (8) the Conjecture 1 and therefore the inclusion (15) are true. Furthermore, the inclusion

$$
C_{d, p}\left(\frac{2}{b(\mathbb{E}(\mathbf{a}))}\right)^{d+1-p} \leq \frac{\mathcal{I}_{p}(\mathbb{E}(\mathbf{a}))}{V^{2}(\mathbb{E}(\mathbf{a}))} \leq C_{d, p}\left(\frac{\kappa_{d}}{V(\mathbb{E}(\mathbf{a}))}\right)^{(d+1-p) / d}
$$

holds with $C_{d, p}=2^{p-1} \pi^{-1 / 2} d \Gamma\left(\frac{d}{2}+1\right) \Gamma\left(\frac{p}{2}+1\right) / \Gamma\left(\frac{d+p+1}{2}\right)$ for any $p \in[1, d]$. For $p=k+1$ we may write $C_{d, k+1}=2^{k} d \kappa_{d+k} / \kappa_{k+1} \kappa_{d}$ for $k=0,1, \ldots, d-1$.

Proof. First we express the ratio $\mathcal{I}_{k+1}(\mathbb{E}(\mathbf{a})) / \mathcal{I}_{k}(\mathbb{E}(\mathbf{a}))$ by means of (10) in terms of the function $Y(\mathbf{u})=1 / h_{\mathbb{E}(\mathbf{a})}(\mathbf{u})$ for $\mathbf{u} \in \mathbb{S}^{d-1}$. Setting $p=k+1$ resp. $p=k$ in (10) and replacing $\Gamma(n / 2+1)$ by $\pi^{n / 2} / \kappa_{n}$ for $n \in\{k, k+1, d-k, d-$ $k+1\}$ yield the identity

$$
\frac{\mathcal{I}_{k+1}(\mathbb{E}(\mathbf{a}))}{\mathcal{I}_{k}(\mathbb{E}(\mathbf{a}))}=\frac{2 \kappa_{k} \kappa_{d+k}}{\kappa_{k+1} \kappa_{d+k-1}} \frac{\int_{\mathbb{S}^{d-1}} Y(\mathbf{u})^{d-k} \mathcal{H}^{d-1}(\mathrm{~d} \mathbf{u})}{\int_{\mathbb{S}^{d-1}} Y(\mathbf{u})^{d-k+1} \mathcal{H}^{d-1}(\mathrm{~d} \mathbf{u})}
$$

The r.h.s. of (18) remains unchanged if $\mathcal{H}^{d-1}(\cdot)$ is replaced by the uniform distribution $U(\cdot):=\mathcal{H}^{d-1}(\cdot) / d \kappa_{d}$ on $\mathbb{S}^{d-1}$. In this way we may write the ratio of the both integrals over $\mathbb{S}^{d-1}$ in (18) as ratio $\mathrm{E}_{U} Y^{d-k} / \mathrm{E}_{U} Y^{d-k+1}$ of moments of the random variable $Y(\cdot)$ w.r.t. the uniform distribution $U$ on $\mathbb{S}^{d-1}$. Putting $k=0$ in (18) yields $\mathcal{I}_{1}(\mathbb{E}(\mathbf{a})) / \mathcal{I}_{0}(\mathbb{E}(\mathbf{a}))=\kappa_{d} \mathrm{E}_{U} Y^{d} / \kappa_{d-1} \mathrm{E}_{U} Y^{d+1}$. Now, we insert this identity in the desired inequality (14) for $K=\mathbb{E}(\mathbf{a})$. Comparing the resulting relation (14) with (18) we deduce that (14) is equivalent with the moment inequality

$$
\frac{\mathrm{E}_{U} Y^{d}}{\mathrm{E}_{U} Y^{d+1}} \leq \frac{\mathrm{E}_{U} Y^{d-k}}{\mathrm{E}_{U} Y^{d-k+1}} \quad \text { for any } \quad k=1,2, \ldots
$$


Since $Y$ is strictly positive and bounded, it easily seen by the Cauchy-Schwarz inequality that

$$
\mathrm{E}_{U} Y^{d-j} \leq \sqrt{\mathrm{E}_{U} Y^{d-j-1}} \sqrt{\mathrm{E}_{U} Y^{d-j+1}} \text { i.e. } \frac{\mathrm{E}_{U} Y^{d-j}}{\mathrm{E}_{U} Y^{d-j+1}} \leq \frac{\mathrm{E}_{U} Y^{d-j-1}}{\mathrm{E}_{U} Y^{d-j}}
$$

for $j=0,1, \ldots, k-1$ and $k=1,2, \ldots$, implying immediately (19). Note that " $="$ holds iff $\mathrm{P}\left(Y=h_{\mathbb{E}(\mathbf{a})}^{-1}=\right.$ const $)=1$, i.e., $a_{1}=\cdots=a_{d}=$ const.

Thus, the first part of Theorem 5.1 is proved. To prove the second part we start with $1=\left(h_{\mathbb{E}(\mathbf{a})}(\mathbf{u}) Y(\mathbf{u})\right)^{1 / 2}$ for $\mathbf{u} \in \mathbb{S}^{d-1}$ and apply the Cauchy-Schwarz and twice the Hölder inequality leading to

$$
1 \leq \mathrm{E}_{U} h_{\mathbb{E}(\mathbf{a})} \mathrm{E}_{U} Y \leq \mathrm{E}_{U} h_{\mathbb{E}(\mathbf{a})}\left(\mathrm{E}_{U} Y^{d+1-p}\right)^{\frac{1}{d+1-p}} \leq \mathrm{E}_{U} h_{\mathbb{E}(\mathbf{a})}\left(\mathrm{E}_{U} Y^{d}\right)^{\frac{1}{d}}
$$

for any real $p \in[1, d]$. Next we write $\mathrm{E}_{U}$ as integral over $\mathbb{S}^{d-1}$ w.r.t. uniform distribution $U$ and make use of the mean breadth $b(\mathbb{E}(\mathbf{a}))=2 \mathrm{E}_{U} h_{\mathbb{E}(\mathbf{a})}$ (see in Sect. 3) with support function (9). This amounts to the inclusion

$$
\left(\frac{2}{b(\mathbb{E}(\mathbf{a}))}\right)^{d+1-p} \leq \int_{\mathbb{S}^{d-1}} \frac{U(\mathrm{~d} \mathbf{u})}{h_{\mathbb{E}(\mathbf{a})}(\mathbf{u})^{d+1-p}} \leq\left(\int_{\mathbb{S}^{d-1}} \frac{U(\mathrm{~d} \mathbf{u})}{h_{\mathbb{E}(\mathbf{a})}(\mathbf{u})^{d}}\right)^{(d+1-p) / d} .
$$

Combining (10) for $p=1$ and $\mathcal{I}_{1}(K)=d \kappa_{d} V(K) / 2$ reveals the remarkable relation

$$
\int_{\mathbb{S}^{d-1}} \frac{U(\mathrm{~d} \mathbf{u})}{h_{\mathbb{E}(\mathbf{a})}(\mathbf{u})^{d}}=\frac{\kappa_{d}}{V(\mathbb{E}(\mathbf{a}))}=\frac{1}{a_{1} \cdots a_{d}} .
$$

We mention that (21) can also be verified directly by using the spherical coordinates $u_{1}=\cos \vartheta_{1} \sin \vartheta_{2} \cdots \sin \vartheta_{d-1}, u_{2}=\sin \vartheta_{1} \sin \vartheta_{2} \cdots \sin \vartheta_{d-1}$ and $u_{i}=\cos \vartheta_{i-1} \prod_{j=i}^{d-1} \sin \vartheta_{j}$ for $i=3, \ldots, d$ with infinitesimal surface element $\mathcal{H}^{d-1}(\mathrm{~d} \mathbf{u})=\prod_{i=2}^{d-1}\left(\sin \vartheta_{i}\right)^{i-1} \mathrm{~d}\left(\vartheta_{1}, \vartheta_{2}, \ldots, \vartheta_{d-1}\right)$, where $\vartheta_{1} \in[0,2 \pi]$ and $\vartheta_{i} \in$ $[0, \pi]$ for $i=2, \ldots, d-1$.

Finally, we multiply the inclusion (20) by the constant $C_{d, p}$ which is chosen in view of (10) such that the middle term multiplied by $C_{d, p}$ just equals $\mathcal{I}_{p}(\mathbb{E}(\mathbf{a})) / V(\mathbb{E}(\mathbf{a}))^{2}$. This completes the proof of Theorem 5.1.

Remark 2. From (17) we get Urysohn's inequality, see [1], for $\mathbb{E}(\mathbf{a})$, namely,

$$
2\left(\frac{V(\mathbb{E}(\mathbf{a}))}{\kappa_{d}}\right)^{1 / d} \leq b(\mathbb{E}(\mathbf{a}))=\frac{2}{d \kappa_{d}} \int_{\mathbb{S}^{d-1}} h_{\mathbb{E}(\mathbf{a})}(\mathbf{u}) \mathcal{H}^{d-1}(\mathrm{~d} \mathbf{u})
$$

with " =" iff $a_{1}=\cdots=a_{d}$. By comparison of the inclusions (15) for $K=\mathbb{E}(\mathbf{a})$ and (17) for $p=k+1$ we see that the upper bounds coincide but the lower bound in (17) for $k=d-1$ (resp. for $k=1$ ) is $\geq$ (resp. $\leq$ ) the lower bound in (15) due to the Aleksandrov-Fenchel-type inequality $2 V(K)(S(K) / d V(K))^{d-1}$ $\geq \kappa_{d} b(K)$ (resp. the isoperimetric-type inequality $\left.b(K) \geq 2\left(S(K) / d \kappa_{d}\right)^{1 /(d-1)}\right)$. 
This gives rise to formulate the following alternative to (15):

Conjecture 2. For any convex body $K \subset \mathbb{R}^{d}$ with inner points and $k \geq 0$,

$$
\frac{2^{k} d}{\kappa_{d}} \frac{\kappa_{d+k}}{\kappa_{k+1}}\left(\frac{2}{b(K)}\right)^{d-k} \leq \frac{\mathcal{I}_{k+1}(K)}{V(K)^{2}} \leq \frac{2^{k} d}{\kappa_{d}} \frac{\kappa_{d+k}}{\kappa_{k+1}}\left(\frac{\kappa_{d}}{V(K)}\right)^{(d-k) / d}
$$

with "=" being attained only for balls, see [6] for the special case $k=d-1$.

\section{CPI's of Superellipsoids and Simplices}

We conclude this paper with a few remarks on CPI's of two important classes of convex bodies in $\mathbb{R}^{d}$ - superellipsoids $\mathbb{E}_{\alpha}(\mathbf{a})$ and simplices $\mathbb{S}(\mathbf{P})$. To be precise, we start with a definition of these bodies. In generalizing (8) the convex body $\mathbb{E}_{\alpha}(\mathbf{a}):=\left\{\mathbf{x} \in \mathbb{R}^{d}:\left(\left|x_{1}\right| / a_{1}\right)^{\alpha}+\cdots+\left(\left|x_{d}\right| / a_{d}\right)^{\alpha} \leq 1\right\}$ is called a superellipsoid $\mathbb{E}_{\alpha}(\mathbf{a})$ of degree $\alpha \geq 1$ with semi-axes $\mathbf{a}=\left(a_{1}, \ldots, a_{d}\right)$ and $\mathbb{S}(\mathbf{P})$ is defined to be the convex hull of the $d+1$ points $\mathbf{P}=\left\{\mathbf{o}, \mathbf{p}_{1}, \ldots, \mathbf{p}_{d}\right\}$, where the vectors $\mathbf{p}_{1}, \ldots, \mathbf{p}_{d}$ are linearly independent.

Special cases are the cross-polytope $\mathbb{E}_{1}(\mathbf{a})$, the ellipsoid $\mathbb{E}(\mathbf{a})$ for $\alpha=2$ and the hyper-rectangle $\mathbb{E}_{\infty}(\mathbf{a})=\times_{i=1}^{d}\left[-a_{i}, a_{i}\right]$ all of them are o-symmetric with support function $\left.h_{\mathbb{E}_{\alpha}(\mathbf{a})}(\mathbf{u})=\left(\left|u_{1}\right| a_{1}\right)^{\beta}+\cdots+\left(\left|u_{d}\right| a_{d}\right)^{\beta}\right)^{1 / \beta}$ with $\beta=\alpha /(\alpha-1)$ for $\alpha>1$ and $h_{\mathbb{E}_{1}(\mathbf{a})}(\mathbf{u})=\max \left\{\left|u_{1}\right| a_{1}, \ldots,\left|u_{d}\right| a_{d}\right\}$.

By introducing the $\ell_{\alpha}$-norm $\|\mathbf{x}\|_{\alpha}=\left(\left|x_{1}\right|^{\alpha}+\cdots+\left|x_{d}\right|^{\alpha}\right)^{1 / \alpha}$ for $1 \leq \alpha<\infty$ and $\|\mathbf{x}\|_{\infty}:=\max \left\{\left|x_{1}\right|, \ldots,\left|x_{d}\right|\right\}$ we define the unit ball $\mathbb{B}_{\alpha}^{d}:=\left\{\mathbf{x} \in \mathbb{R}^{d}:\|\mathbf{x}\|_{\alpha} \leq 1\right\}$ and its volume $\kappa_{d, \alpha}:=V\left(\mathbb{B}_{\alpha}^{d}\right)$ w.r.t. the $\ell_{\alpha}$-norm. Since $\mathbb{E}_{\alpha}(\mathbf{a})=A \mathbb{B}_{\alpha}^{d}$ with diagonal matrix $A=\operatorname{diag}\left[a_{1}, \ldots, a_{d}\right]$ it follows that $V\left(\mathbb{E}_{\alpha}(\mathbf{a})\right)=\kappa_{d, \alpha} \prod_{i=1}^{d} a_{i}$. By repeating the proof of Theorem 1 (which is left to the reader) we can generalize (10) as follows:

Theorem 6.1 It holds that $\mathcal{I}_{1}\left(\mathbb{E}_{\alpha}(\mathbf{a})\right)=\frac{d}{2} \kappa_{d} \kappa_{d, \alpha} \prod_{i=1}^{d} a_{i}$ and, for $p>1$,

$$
\frac{\mathcal{I}_{p}\left(\mathbb{E}_{\alpha}(\mathbf{a})\right)}{V^{2}\left(\mathbb{E}_{\alpha}(\mathbf{a})\right)}=\frac{p(p-1)}{2 \kappa_{d, \alpha}^{2}} \int_{\partial \mathbb{B}_{\gamma}^{d}} \int_{0}^{2 /\|\mathbf{u}\|_{\alpha}} \frac{V\left(\mathbb{B}_{\alpha}^{d} \cap\left(\mathbb{B}_{\alpha}^{d}+r \mathbf{u}\right)\right) r^{p-2}}{h_{\mathbb{E}(\mathbf{a})}(\mathbf{u})^{d+1-p}} \mathrm{~d} r \mathcal{H}^{d-1}(\mathrm{~d} \mathbf{u})
$$

for arbitrary $\gamma \in[1, \infty]$, where $2 /\|\mathbf{u}\|_{\alpha}=\inf \left\{r>0: \mathbb{B}_{\alpha}^{d} \cap\left(\mathbb{B}_{\alpha}^{d}+r \mathbf{u}\right)=\emptyset\right\}$.

To conclude with, we quote a result on CPI's of $\mathbb{S}(\mathbf{P})$ proved in [15] (p.593)

$$
\frac{\mathcal{I}_{p}(\mathbb{S}(\mathbf{P}))}{V(\mathbb{S}(\mathbf{P}))}=\frac{d ! \Gamma(p+1)}{2 \Gamma(p+d)} \int_{\mathbb{S}^{d-1}}\left(\frac{d V(\mathbb{S}(\mathbf{P}))}{\mathcal{H}^{d-1}\left(\mathbb{S}(\mathbf{P}) \mid \mathbf{u}^{\perp}\right)}\right)^{p-1} \mathcal{H}^{d-1}(\mathrm{~d} \mathbf{u})
$$


for any $p \geq 1$ with the well-known formula $V(\mathbb{S}(\mathbf{P}))=\left|\operatorname{det}\left(\mathbf{p}_{1}, \ldots, \mathbf{p}_{d}\right)\right| / d !$.

An astonishing relation proved in [8] says that $\mathcal{H}^{d-1}\left(\mathbb{S}(\mathbf{P}) \mid \mathbf{u}^{\perp}\right) \rho_{D \mathbb{S}(\mathbf{P})}(\mathbf{u})=$ $d V(\mathbb{S}(\mathbf{P}))$ for any $\mathbf{u} \in \mathbb{S}^{d-1}$, where $\rho_{D K}(\mathbf{u}):=\max \{\lambda>0: \lambda \mathbf{u} \in K \oplus(-K)\}$ denotes the radial function of the difference body $D K:=K \oplus(-K)$ of $K$. It is not difficult to show that $\rho_{D K}(\mathbf{u})=\max \left\{\mathcal{H}^{1}(K \cap \ell(\mathbf{x}, \mathbf{u})): x \in \mathbf{u}^{\perp}\right\}=$ length of the longest chord of $K$ in direction $\mathbf{u}$. In view of this geometric relation the integrand on the r.h.s. of (23) can be replaced by $\rho_{D \mathbb{S}(\mathbf{P})}(\mathbf{u})^{p-1}$, see also [2] for a different approach.

\section{References}

[1] T. Bonnesen und W. Fenchel, Theorie der konvexen Körper, (corrected reprint of first edn. in 1934), Springer, Berlin, 1974.

[2] W.S. Cheung and G. Xiong, Chord power integrals of simplices, Asian-European J. of Math., 2 (2009), 557 - 566.

[3] P.J. Davy, Inequalities for moments of secant length, Z. Wahrscheinlichkeitstheorie verw. Gebiete, 68 (1984), 243 - 246.

[4] W. Gille, Particle and Particle Systems Characterization - Small-Angle Scattering (SAS) Applications, CRC Press, Boca Raton, 2014.

[5] J. Hansen and M. Reitzner, Electromagnetic wave propagation and inequalities for moments of chord lengths, Adv. Appl. Prob.,36 (2004), 987 - 995.

[6] L Heinrich, On lower bounds of second-order chord power integrals of convex discs, Rend. Circ. Mat. Palermo, Serie II, Suppl.,81 (2009), 213 - 222.

[7] L. Heinrich and M. Spiess, CLT for volume and surface content of stationary Poisson cylinder processes in expanding domains, Adv. Appl. Prob.,45 (2013), $312-331$.

[8] H. Martini and B. Weissbach, On quermasses of simplices, Studia Scientiarum Mathematicarum Hungarica,27 (1992), 213-221.

[9] F. Piefke, Beziehungen zwischen der Sehnenlängenverteilung und der Verteilung des Abstandes zweier zufälliger Punkte im Eikörper, Z. Wahrscheinlichkeitstheorie verw. Gebiete,43 (1978), 129 - 134.

[10] F. Piefke, Chord length distribution of the ellipse, Lithuanian Math. J.,19 (1979), $325-333$.

[11] L.A. Santaló, Integral Geometry and Geometric Probability, Addison-Wesley, Reading (Mass.) 1976. 
[12] R. Schneider and J.A. Wieacker, Integral Geometry. In Handbook of Convex Geometry, eds. P.M. Gruber, J.M. Wills, Vol. B, 1345 - 1390, North-Holland, Amsterdam 1993.

[13] R. Schneider and W. Weil, Stochastic and Integral Geometry. Springer, Berlin 2008 .

[14] K. Voss, Powers of chords for convex sets, Biom. J.,24 (1982), 513 - 516.

[15] G. Xiong and X. Song, Inequalities for chord power integrals, J. Korean Math. Soc.,45 (2008), 587 - 596.

Received: September 1, 2014; Published: November 25, 2014 